\title{
Scale-Up of Magnetocaloric NiCoMnIn Heuslers by Powder Metallurgy for Room Temperature Magnetic Refrigeration
}

\begin{abstract}
Francesco Puglielli ${ }^{1}$, Valerio Mussi ${ }^{2}$, Francesco Cugini ${ }^{3,4}$, Nicola Sarzi Amadì ${ }^{3,4}$, Massimo Solzi ${ }^{3,4}$, Cecilia Bennati ${ }^{4}$, Simone Fabbrici ${ }^{4,5 *}$ and Franca Albertini ${ }^{4}$

${ }^{1}$ Department of Mechanical Engineering, Polytechnic University of Milan, Milan, Italy, ${ }^{2}$ MUSP, Machine Tools and Production Systems Laboratory, Piacenza, Italy, ${ }^{3}$ Department of Mathematical, Physical and Computer Sciences, University of Parma, Parma, Italy, ${ }^{4}$ Institute of Materials for Electronics and Magnetism - National Research Council Institute, Parma, Italy, ${ }^{5}$ MIST E-R, Micro and Submicro Technologies for Emilia Romagna, Bologna, Italy
\end{abstract}

We present a new approach for a large-scale production of the rare-earth free NiCoMnln Heusler alloy for room temperature magnetic refrigeration applications. This class of compounds has recently attracted attention, thanks to the large reversible isothermal entropy change $\left(\Delta S_{\text {iso }}\right)$ and adiabatic temperature change $\left(\Delta T_{\mathrm{ad}}\right)$ associated to a first-order magnetostructural phase transition. A large-scale production method, however, has not yet been proposed. For giant magnetocaloric materials and especially for Heusler compounds, the synthesis has a predominant role in tailoring the physical-chemical properties, due to the high sensitivity of the first-order transition characteristics on chemical composition and microstructure. Up to $250 \mathrm{~g}$ of the nominal composition $\mathrm{Ni}_{45.7} \mathrm{Co}_{4.2} \mathrm{Mn}_{36.6} \mathrm{In}_{13.3}$ alloy was prepared in a unique sample starting from industrial-grade powdered elements. The phase transition temperatures and magnetocaloric properties were investigated by magnetic and direct adiabatic temperature measurements and were found to be homogeneous in the whole sample. The mechanical stability of the produced alloy and its workability were investigated. A low-temperature thermal treatment was identified and showed promising results by reducing hysteresis and transition width.

Specialty section:

This article was submitted to Process and Energy Systems Engineering,

a section of the journal Frontiers in Energy Research

Received: 01 November 2019 Accepted: 06 December 2019 Published: 10 January 2020

Citation:

Puglielli F, Mussi V, Cugini F Sarzi Amadè N, Solzi M, Bennati C, Fabbrici S and Albertini F (2020)

Scale-Up of Magnetocaloric NiCoMnIn Heuslers by Powder Metallurgy for Room Temperature

Magnetic Refrigeration

Front. Energy Res. 7:150. doi: 10.3389/fenrg.2019.00150
Keywords: Heusler alloys, magnetocaloric effect, magnetic refrigeration, scale-up, large-scale synthesis, powder metallurgy, martensitic transformation

\section{INTRODUCTION}

The mass-market introduction of affordable refrigeration technology has shaped the development of society and improved everyday life over the last century. Current estimates show that the amount of electricity consumed worldwide by refrigeration and air conditioning is increasing yearly and has now reached 20\% (International Institute on Refrigeration IIR_IIF, 2019). The physical principle at the basis of the current cooling technology is the compression-expansion cycle of the refrigerant gases, which has the benefit of being cheap and effective, yet such gases have the very important drawback of contributing to the global warming due to their consistent greenhouse effect as they leak in the atmosphere when the refrigerators are disposed of (Gauss et al., 2017).

Solid-state cooling technologies based on caloric effects, such as magnetocaloric (MCE), barocaloric, elastocaloric, electrocaloric, and coupled multicaloric, are emerging alternatives to the 
vapor compression technology. Among them, magnetocaloric refrigeration is the most mature: since the first demonstration of room temperature magnetocaloric refrigeration and the discovery of the first giant magnetocaloric alloy in the mid-1990s (Pecharsky and Gschneidner, 1997; Zimm et al., 1998), several tens of prototypes have been presented and demonstrated the great potential for applications of this new technology (Kitanovski et al., 2015).

Magnetocaloric materials are the core of the magnetic refrigerators. They are magnetic materials with magnetic phase transitions around room temperature; when exposed to a magnetic field change in a temperature range close to the transition temperature, they respond by heating and cooling by a few degrees. Such temperature change is exploited in a regenerative thermal cycle that allows to produce a temperature span between the hot and cold exchangers of a magnetocaloric heat pump that can be several times larger than the adiabatic temperature change of the material, ultimately enabling the application. The temperature change at the magnetocaloric transition grows with the intensity of the magnetic field change; yet, to build energetically efficient devices for room temperature applications, the magnetic field is limited in magnitudes to the values achievable by permanent magnets, typically ranging around $1-1.5 \mathrm{~T}$.

The operational range and the requirements imposed by the efficiency considerations set the desirable materials properties for room temperature refrigeration, namely, magnetic transition close to room temperature and easily tunable by composition, high and reversible temperature changes over a temperature span suitable for a thermal cycle (i.e., of a few tens of degrees), complete reversibility of the transition in magnetic fields produced by permanent magnets, and mechanical properties suitable for achieving regenerators with high surface/volume ratios (e.g., packed submillimeter spheres or thin plates).

Several classes of magnetic materials have been extensively studied in the last decades searching for good candidates of magnetocaloric coolants for room temperature application. Magnetic elements and compounds showing either secondorder Curie transitions or first-order magnetostructural transformations have been proposed, such as gadolinium, $\mathrm{Gd}-\mathrm{Si}-\mathrm{Ge}, \mathrm{La}(\mathrm{Fe}, \mathrm{Si})_{13}$, Ni-Mn-based full Heusler compounds $(\mathrm{MnFe})_{2}$ P-type compounds, and La,Ca-based manganites (Franco et al., 2018). Stoichiometry has been a powerful tool for tuning the working temperature of these materials and for improving the size of the magnetocaloric effect.

To date, a couple of materials have been extensively tested in refrigerator prototypes. Gadolinium is the reference magnetocaloric coolant: it shows a completely reversible and quite consistent magnetocaloric effect (adiabatic temperature change, $\Delta T_{\text {ad }}=2.5 \mathrm{~K}$ in $1 \mathrm{~T}$ ) at its Curie point, which spans for a few tens of degrees. As it is an element, it is easy to achieve reproducible results, as long as it is used over a certain degree of purity. The first commercial material besides gadolinium is the $\mathrm{La}(\mathrm{FeSi})_{13}$ family of compounds. Since its magnetocaloric effect originates from a first-order transition, the temperature range where a useful temperature change is produced is much more limited than the Gd one: this feature adds complexity to the machine design, as it requires a cascade-type regenerator where several sections with carefully tuned compositions and therefore transition temperatures are employed, the malfunction of one element jeopardizing the entire thermal cycle.

Looking into the perspective of technological deployment, many critical issues have to be overcome, both from the point of view of machining and magnetocaloric performances and from the point of view of materials criticality. Gadolinium, for example, is considered a critical material in terms of geopolitical availability: this may represent a potential issue for a future mass-market technology (Gauss et al., 2017).

Among other classes of materials suitable for the magnetocaloric refrigeration, the Ni-Mn-based Heusler compounds are one of the most promising. This family of compounds includes many different kinds of compositions, making use of a large variety of elements, such as manganese, nickel, cobalt, gallium, tin, aluminum, indium, and others (Aksoy et al., 2009). They display a second-order Curie transition and a first-order magnetostructural transformation that can be easily tuned with composition both in terms of critical temperatures and in terms of entity and sign of the magnetocaloric effect (Pareti et al., 2003; Fabbrici et al., 2014; Cavazzini et al., 2019). Up to now, the quaternary system Ni-Co-Mn-In has shown the highest values, among all Heuslers, of maximum and reversible adiabatic temperature change in a 2-T field (Gottschall et al., 2015) associated to the first-order martensitic transformation: $\Delta T_{\mathrm{ad}}$ values of $8 \mathrm{~K}$ (peak) and $3 \mathrm{~K}$ (reversible) for a 2-T field change measured for sample $\mathrm{Ni}_{45.7} \mathrm{Co}_{4.2} \mathrm{Mn}_{36.6} \mathrm{In}_{13.3}$ (Gottschall et al., 2015) are close to the performance of gadolinium.

Magnetocaloric Heusler compounds are usually obtained through melting the single elements; many different techniques can be exploited for their production on a laboratory scale. Polycrystals, indeed, can be produced much more easily with respect to single crystals (Feng et al., 2018) and are mostly obtained through arc melting (Sánchez-Alarcos et al., 2008; Feng et al., 2009; Chen et al., 2010, 2011; Gottschall et al., 2015) or induction melting (Kainuma et al., 2006; Ito et al., 2008; Liu et al., 2012; Bruno et al., 2018). High-purity precursors in the form of lumps, flakes, and chips are generally melted together in a protected environment (argon atmosphere) to obtain few grams of the alloy. Successive thermal treatments are needed to obtain a satisfying homogeneity and a single-phase alloy with the required magnetocaloric properties. Unfortunately, the final material is often characterized by low mechanical properties and severe brittleness, which can compromise its employment in a working device. Melt spinning is also a well-known technique that is used to produce highly textured materials thanks to a very rapid quenching. In addition, the final annealing is generally not required to obtain a single-phase alloy. However, melt-spun ribbons are yet again very small and extremely brittle (Feng et al., 2018).

Spark plasma sintering is a technique recently used to obtain bulky Heusler compounds, which consists in heating the sample with pulsed dc current maintaining the powder under pressure. This technique can produce textured materials with good mechanical properties starting from powdered alloy (Ito et al., 2009) or different shapes. The same technique has been 
employed, for instance, to sinter together several melt-spun ribbons controlling the grains orientation and improving the ribbons' mechanical properties (Feng et al., 2018).

Microwaves-assisted sintering was also recently proposed to prepare Heusler compounds starting form elemental powders (Trombi et al., 2020), but the use of this technique for the preparation of off-stoichiometric martensitic compositions has not yet been optimized.

Generally, both brittleness, and small sample mass can be considered as major obstacles for further development of the materials, in terms of structural reliability and workability. In this sense, the possibility to produce dense samples with reduced brittleness and significant dimensions is a mandatory step that still must be conquered. Three typical designs for active magnetic regenerators are generally considered (Waske et al., 2018): packed beds (made of irregularly shaped powders or spherical particles), stacked thin plates, and microchannels geometries. A common strategy for packed beds and thin plates to increase the mechanical reliability is to use a polymeric reinforcement. Problems like active magnetic regenerator cracking and device failure can be limited in this way. However, the heat exchange capabilities will be restricted, and the active material allowed in the device's volume will be reduced. In the case of microchannel geometries instead, the most important problem is still the mechanical behavior; however, the difficulty in having a reliable and cost-effective industrial process must also be considered.

In short, a large-scale and reliable production method of magnetocaloric Heusler compounds scalable for a future worldwide market has not yet been demonstrated.

In this work, we focused on the reliable and largescale production of polycrystalline $\mathrm{Ni}_{45.7} \mathrm{Co}_{4.2} \mathrm{Mn}_{36.6} \mathrm{In}_{13.3}$ alloy. This specific composition was chosen due to the promising magnetocaloric properties reported in the literature (Gottschall et al., 2015). We propose a new approach to increase the mechanical stability of Heusler compounds and to obtain a scalable, cost-effective process. The samples were prepared using industrial-grade powdered elements to reduce the costs of the experimental campaign and to foresee a market-oriented standard. The main physical properties of interest have been characterized: composition, structure, magnetic, magnetocaloric, and mechanical properties. Suitable thermal treatments to reduce the hysteretic behavior are proposed.

\section{MATERIALS AND METHODS}

\section{Samples Preparation}

The $\mathrm{Ni}_{45.7} \mathrm{Co}_{4.2} \mathrm{Mn}_{36.6} \mathrm{In}_{13.3}$ alloy samples were produced starting from industrial-grade powdered elements. The purity grades and particle sizes of the reagents are listed in the Table $\mathbf{1}$. Each constituent was weighted in the stoichiometric quantity and mixed with all the others using a planetary mixer for $1 \mathrm{~h}$. The final mix was pressed into pellets to obtain a compact precursor by applying for $30 \mathrm{~s} 300 \mathrm{MPa}$ with a cylindrical die and a SACMI industrial press. This procedure allows for the production of precursors with any value of mass and shape factor. Many samples were prepared to optimize the technique, starting from $5 \mathrm{~g}$ and up to $125 \mathrm{~g}$, cylindrical.
TABLE 1 | Characteristics of the powder reagents used to obtain the compacted precursors.

\begin{tabular}{|c|c|c|c|c|}
\hline & $\mathbf{N i}$ & Mn & In & Co \\
\hline $\begin{array}{l}\text { Purity grade } \\
(\%)\end{array}$ & 99.9 & 99.7 & 99.9 & 99.5 \\
\hline $\begin{array}{l}\text { Dimension } \\
(\mu \mathrm{m})\end{array}$ & $>63$ (mesh 230) & $<45$ (mesh 325) & $<57$ (mesh250) & $<37$ (mesh 400) \\
\hline
\end{tabular}

The samples were synthesized following two routes, named $\mathrm{A}$ and $\mathrm{B}$, whose temperature-time diagram are depicted in Figure 1. The main difference between the two groups of samples lies in the number of meltings during the preparation: group A samples where melted once, while group B samples were melted twice. Here is the detailed description of the two synthesis protocols. The first melting step, common to both routes, was carried out using an alumina tube furnace in argon atmosphere; each sample was melted in an Alumina crucible. The maximum temperature of $1,773 \mathrm{~K}$ was reached with a heating ramp of $180 \mathrm{~K} / \mathrm{h}$ and maintained for $1 \mathrm{~h}$. This temperature was selected to ensure a complete melting of all the constituents into a low-viscosity fluid. The maximum cooling ramp feasible with this setup was $\sim 200 \mathrm{~K} / \mathrm{h}$ until reaching $573 \mathrm{~K}$; then, only natural cooling was possible. Samples of group A were subsequently heat treated (homogenization step) using an electric muffle furnace for 3 days at $1,188 \mathrm{~K}$ in a protective argon atmosphere and then quenched into water.

Since the slow cooling ramp of the first melting step can negatively affect the microstructure, the second group of specimens, group B, was melted twice before the homogenization step. The first melting step is common to the group A samples; the second melting was carried out using the electric muffle furnace instead of the tube furnace used in the first step. After the first melting, the group B samples, still in the native crucibles, were sealed inside an inox steel box to ensure an inert argon atmosphere. The argon pressure inside the vessel at ambient temperature was $\sim 0.2$ bar. These samples were rapidly heated to $1,373 \mathrm{~K}(75 \mathrm{~K} / \mathrm{min})$. After $15 \mathrm{~min}$, the sealed box was extracted and cooled down to $\sim 1,123 \mathrm{~K}$ by natural cooling (around $2 \mathrm{~min}$ ); once this temperature was reached, the sealed box was put back inside the furnace for the homogenization step with the same parameters followed for group A and finally water quenched. This procedure was used to establish a different microstructure with respect to the group A samples.

Three different samples are considered in the present work: a $50 \mathrm{~g}$ sample melted only one time named A50; a 50 and a $250 \mathrm{~g}$ samples melted two times and named B50 and B250. All three samples were studied for what concern the magnetocaloric properties, while only A50 and B250 samples were considered for testing the mechanical properties.

The impact of a secondary low temperature annealing was then performed on small pieces derived from sample A50. Such $3 \times 3 \times 2 \mathrm{~mm}^{3}$ pieces were cut out from the bulk with an IKC IDRO waterjet machine. The waterjet cut was carried out using 

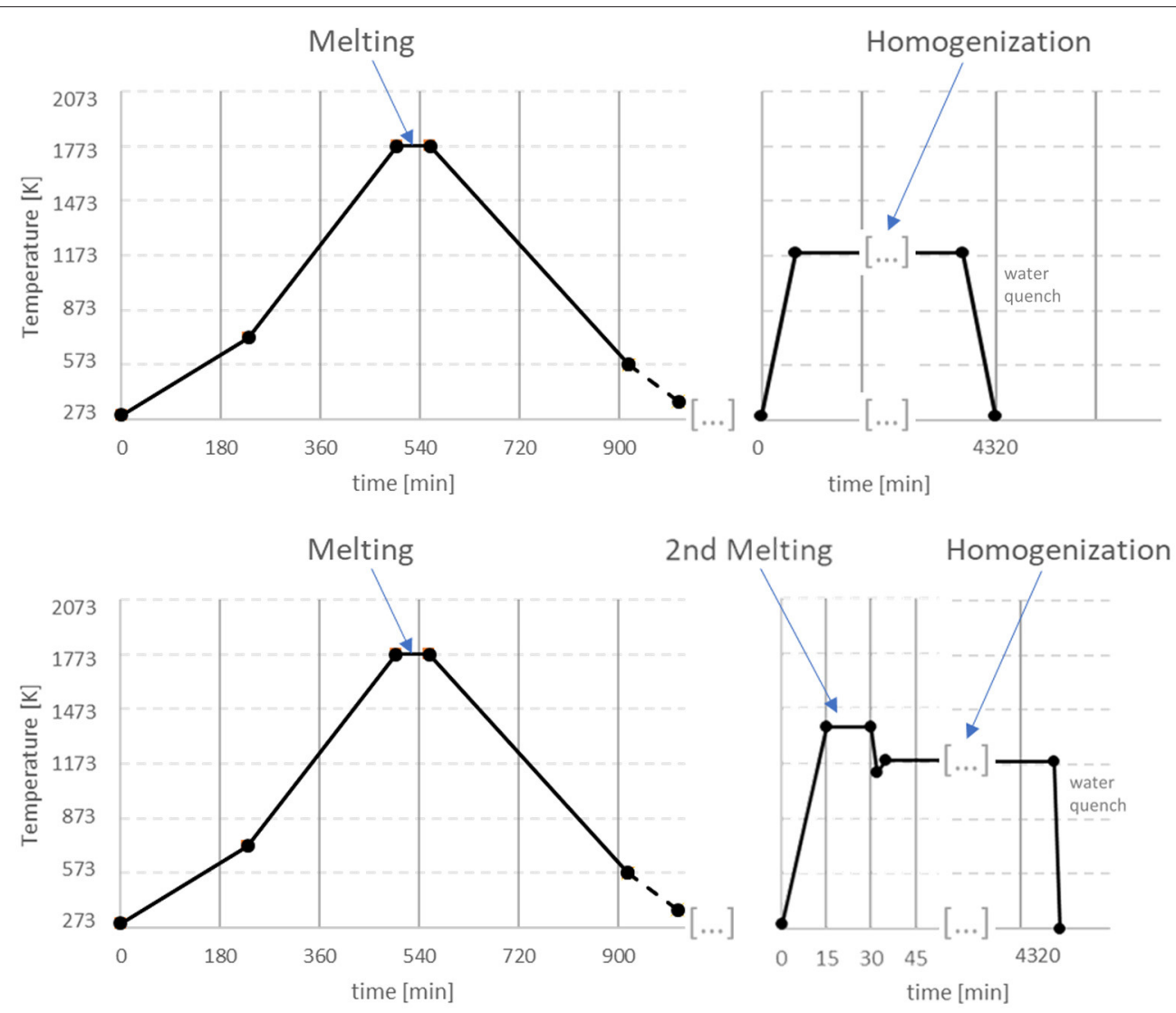

FIGURE 1 | Cartoon summarizing the synthesis protocols for group A (top panel) and group B (bottom panel) samples. Group A samples were melted once; group B samples were melted twice.

the following parameters: 3,000 bar water pressure, $50 \mathrm{~mm} / \mathrm{min}$ cutting speed, and $400 \mathrm{~g} / \mathrm{min}$ of a Garnet abrasive mesh 80 .

The selected temperature for the secondary annealing was 523 K. Sample A50_1 was treated for $1 \mathrm{~h}$, A50_6 for $6 \mathrm{~h}$, and A50_24 for $24 \mathrm{~h}$. All the synthesis conditions for the presented samples are summarized in Table 2. The small samples were introduced into the already hot muffle furnace, so that the heating ramp was the fastest possible $(\sim 125 \mathrm{~K} / \mathrm{min})$. All the samples were water quenched after the treatment to ensure a fast cooling ramp as well.

\section{Composition, Structure, and Mechanical Properties Characterization}

The final chemical composition of the produced samples was determined using a Zeiss EVO 50 scanning electron microscope (SEM) equipped with an X-ray energy dispersive spectroscopy microanalysis probe.

The crystal structure was measured for both the martensitic and the austenitic phases by means of temperature dependent X-ray diffraction collected with a Thermo X'tra powder Xray diffractometer equipped with an environmental chamber. Several diffraction patterns at temperatures below and above the martensitic transformation were collected.
TABLE 2 | Synthesis protocols applied to the samples reported in this work.

\begin{tabular}{lccc}
\hline Sample & 1st fusion & 2nd fusion & Annealing \\
\hline A50 & $1,773 \mathrm{~K}, 1 \mathrm{~h}$ & - & $1,188 \mathrm{~K}, 72 \mathrm{~h}$ \\
B50 & $1,773 \mathrm{~K}, 1 \mathrm{~h}$ & $1,373 \mathrm{~K}, 15 \mathrm{~min}$ & $1,188 \mathrm{~K}, 72 \mathrm{~h}$ \\
B250 & $1,773 \mathrm{~K}, 1 \mathrm{~h}$ & $1,373 \mathrm{~K}, 15 \mathrm{~min}$ & $1,188 \mathrm{~K}, 72 \mathrm{~h}$ \\
\hline Sample & 2nd annealing on sample A50 (temperature, time) \\
\hline A50_1 & $523 \mathrm{~K}$ & $1 \mathrm{~h}$ & \\
A50_6 & $523 \mathrm{~K}$ & $6 \mathrm{~h}$ & \\
A50_24 & $523 \mathrm{~K}$ & $24 \mathrm{~h}$ &
\end{tabular}

The temperatures (in K) and durations (in h) of the various treatments are specified.

A digital microscope VHX 7000 by Keyence was used to scan the surface morphology of the different samples.

A Kern AES 220-4 density measurements kit was used to define the density of three different specimens obtained from A50 and B250 samples.

The Vickers hardness of the samples A50 and B250 was measured following the ISO 14577 standard procedure using a microhardness tester (Fischerscope HM2000 by Fisher). The indentation modulus $E_{\mathrm{IT}}(\mathrm{GPa})$ and $\eta_{\mathrm{IT}}$, i.e., the ratio between 
TABLE 3 | Summary of the average, surface, and bottom chemical compositons for the A50, B50, and B250 samples.

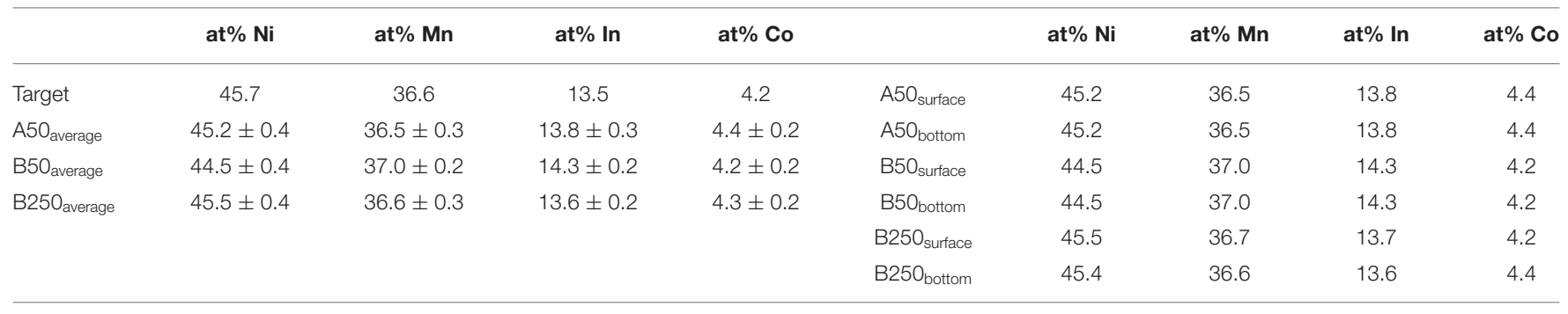

the elastic reverse deformation work of indentation $\left(W_{\text {elast }}, \mathrm{Nm}\right)$ and the total mechanical work of indentation $\left(W_{\text {tot }}, \mathrm{Nm}\right)$ were also measured with the same instrument. All the tests have been performed in a metrology room, with controlled temperature of $298 \mathrm{~K}$.

\section{Magnetocaloric Characterization}

The magnetic properties of prepared specimens were investigated by a magnetic susceptometer, working between 100 and $800 \mathrm{~K}$ with an ac magnetic field of $0.5 \mathrm{mT}$ at $500 \mathrm{~Hz}$, an extraction magnetometer (MagLab System2000 by Oxford Instruments), and a Superconducting Quantum Interference Device magnetometer (Quantum Design MPMS-XL5). The magnetic measurements were used to determine the magnetocaloric entropy change by indirect methods. On the other hand, the adiabatic temperature change was directly measured with a homemade experimental setup based on a Cernox bare chip temperature sensor (Porcari et al., 2013).

\section{RESULTS AND DISCUSSION}

\section{Composition, Structure, and Mechanical Properties}

For both groups A and B, energy-dispersive X-ray analysis confirmed that the average chemical composition is very close to the target one. The atomic compositions are shown in the Table 3. In addition, there is a very good homogeneity within each sample, with no evidence of stratification due to the gravity effect or preferential solidification phenomena. These results represent a strongpoint of our synthesis protocols: in fact, standard melting of compacted powders in alumina crucibles can easily give rise to composition gradients in samples thicker than $10 \mathrm{~mm}$.

The structural characterization of the samples has been performed by means of temperature-dependent powder X-ray diffraction. Patterns at both above and below the structural transition temperatures have been collected for all samples of both $\mathrm{A}$ and $\mathrm{B}$ series, showing the reversible transition from cubic austenite to a low-symmetry martensitic phase, which is compatible with a $7 \mathrm{M}$ modulated monoclinic type of structure typical of many Ni-Mn-based Heuslers (Righi et al., 2011). No impurity phases have been detected with these measurements. The diffraction patterns of sample B50, which is representative of all the series of samples, are reported in Figure 2.

Despite a very similar composition and structural evolution among all the samples, the initial morphological analysis

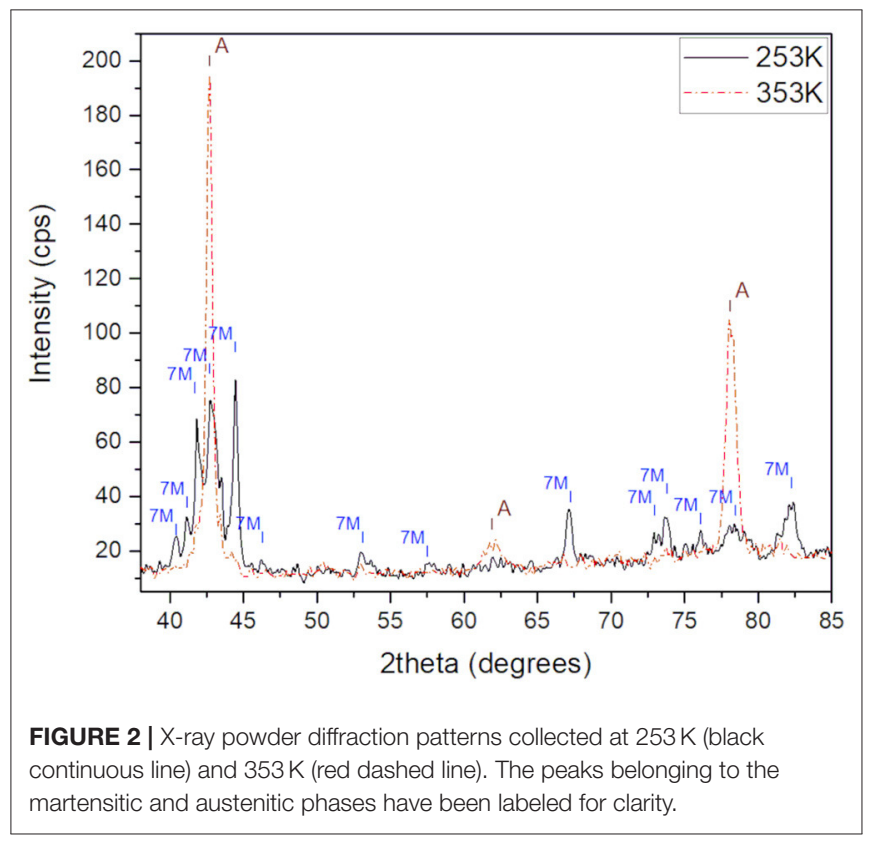

performed by optical microscopy (Figure 3) shows a striking difference between the two groups. All the panels in Figure 3 show the surface of two samples with the same magnification (the $1 \mathrm{~mm}$ marker is reported in all the panels). The left panels (top and bottom) show two regions from sample A50, while the right panels (top and bottom) show two regions from sample B250. The images in the top panels of Figure 3 have been collected with full ring illumination, which provides higher light flux on the sample. The bottom panels images were collected in a coaxial light setup, which provides better visualization of surface textures such as pores and dendrites.

Group A samples (Figure 3, left panels) showed widespread dendritic microstructure, which likely forms during the very slow cooling rate from the molten state at the end of the first fusion. The main drawbacks of such dendrites are the generation of brittleness and friability, features which could occasionally be observed during the samples handling and cutting of parts for measuring purposes. In addition, dendrites could be sites of possible local compositional inhomogeneity due to element segregation.

On the other hand, by introducing the second melting, no dendrites were found within the specimens of group B but a very compact bulk (right panels of Figure 3). This improvement 


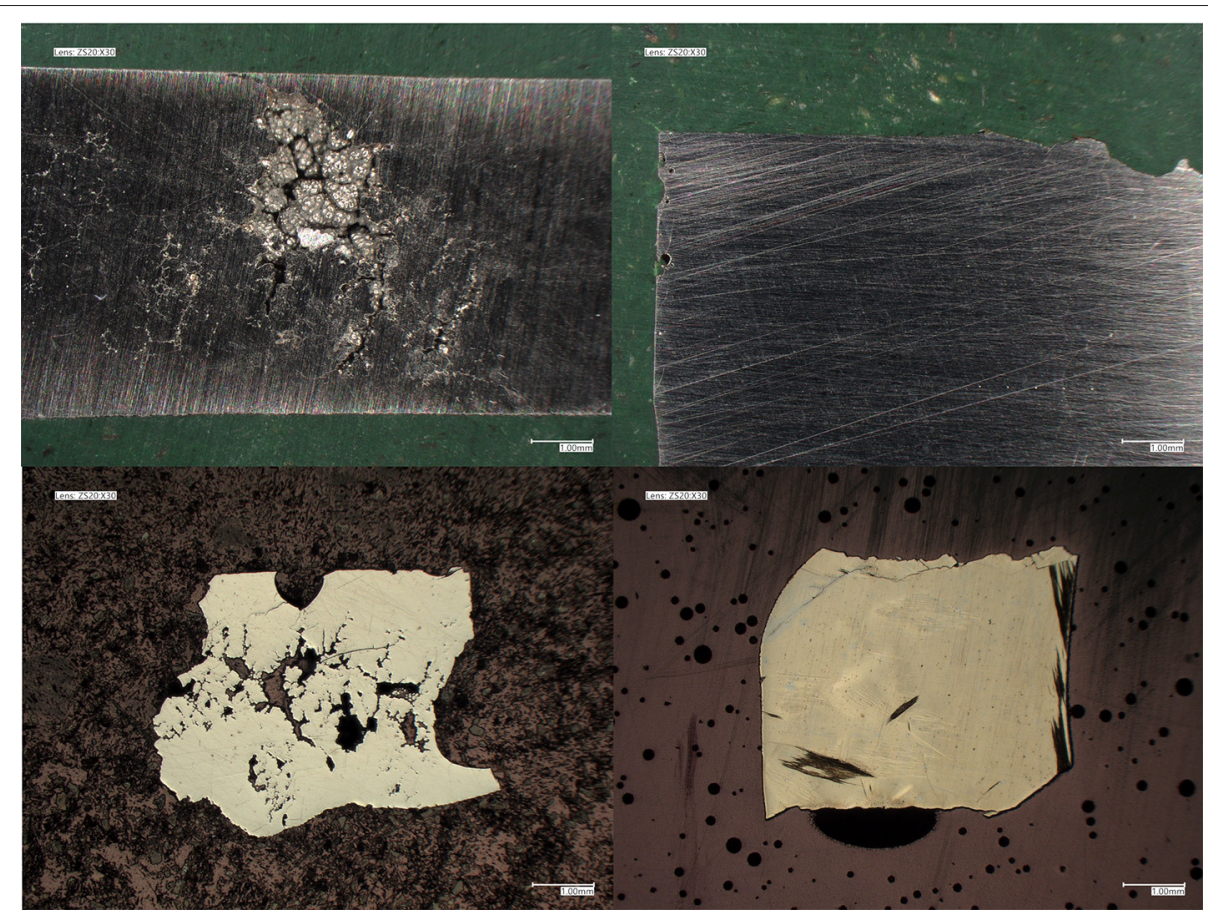

FIGURE 3 | Top and bottom left panels: examples of widespread dendrites and interconnected pores on a polished A50 sample. Top and bottom right panels: absence of dendrites and minor pores appearing on a polished B250 sample.

can be attributed to the double-melting protocol: the dendrites obtained during the first melting are dissolved and cannot be formed again because of the fast cooling provided by the second melting. The mechanical processing performed on group B samples, done by waterjet or diamond blade and polishing, were comparatively simple. On the other hand, group A specimens repeatedly show unwanted cracks, sudden ruptures, and loss of small particles.

Density measurement for two representative samples of groups A and B were carried out by Archimedes' method in water and are reported in Table 4. A vacuum pump was used to allow the water to fill all the interconnected pores present in the samples. Dendrites in the group A samples form extensive interconnected porosity, whereas in the group B samples, only occasional and isolated pores were observed: this is also confirmed by the similar values of density (within the experimental error) measured for samples A50 and B250.

The measurements of Vickers hardness HV, indentation modulus $E_{\mathrm{IT}}$, and the fraction of the elastic reverse deformation work of indentation $\eta_{\text {IT }}$ are also reported in Table 4 . The Vickers hardness, which is partially influenced by extrinsic features such as different microstructures, is slightly higher in the group A sample. On the other hand, both $E_{\mathrm{IT}}$ and $\eta_{\mathrm{IT}}$ show smaller deviations and are considered not statistically different. These results can be interpreted by considering that, contrary to the Vickers hardness, the latter quantities are linked to the intrinsic properties such as crystal structure and composition rather than extrinsic features like microstructure and are therefore consistent with the homogeneous results found by the compositional and structural characterization presented above. It is important to point out that the indenter imprint used for these measures has a surface area which is roughly 2 orders of magnitude smaller than a dendrite tip; this feature allows to precisely probe the materials in morphologically homogeneous areas and therefore to perform proper comparisons among the two samples. By repeating the measures on several positions throughout the samples, it has been possible to evaluate the standard deviations for all the quantities of interest. As evidenced in Table 4, all the measured values show consistently higher standard deviations (2- to 3-fold higher) for the A50 sample with the dendritic microstructure, testifying the lower homogeneity of the mechanical properties of the group A samples. These results are an additional indication that the double-melting process performed on group B, together with the faster cooling rates, improves the homogeneity of the mechanical properties throughout the sample and suggest an easier processing for the latter materials.

\section{Magnetic and Magnetocaloric Properties}

The measurement of the temperature evolution of the magnetic properties shows that all the investigated samples (A50, B50, and B250) possess two magnetic transitions relevant to the magnetocaloric application: a magnetostructural transformation from a low-moment martensite to the ferromagnetic austenite, between 280 and $300 \mathrm{~K}$, and the Curie transition of the austenitic phase $\sim 390 \mathrm{~K}$. Figure 4, top panel presents the magnetization of the samples as a function of temperature measured on heating and on cooling under an applied magnetic field of 1.0 T. The three samples undergo a magnetostructural transition from a very low-moment martensite $\left(M<2 \mathrm{Am}^{2} \mathrm{~kg}^{-1}\right.$ in a magnetic field $\mu_{0} \mathrm{H}=1 \mathrm{~T}$ ) to a ferromagnetic austenite, characterized 
TABLE 4 | Summary of the measured mechanical properties for A50 and B250; meanvalues, standard deviations, and number of readings are displayed.

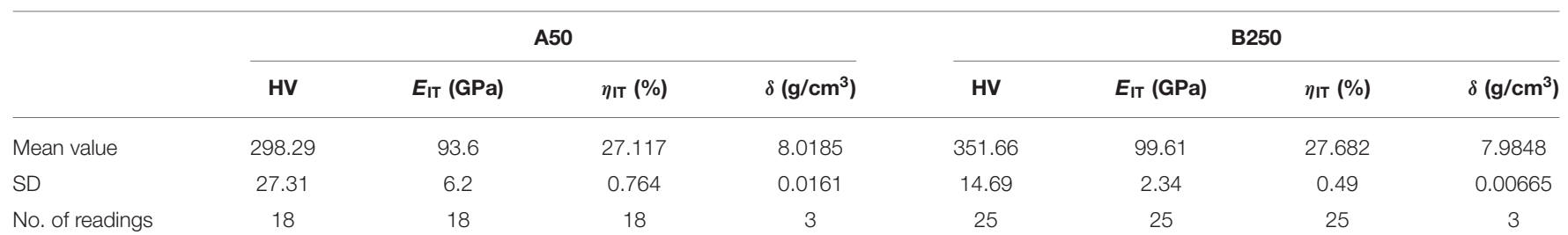

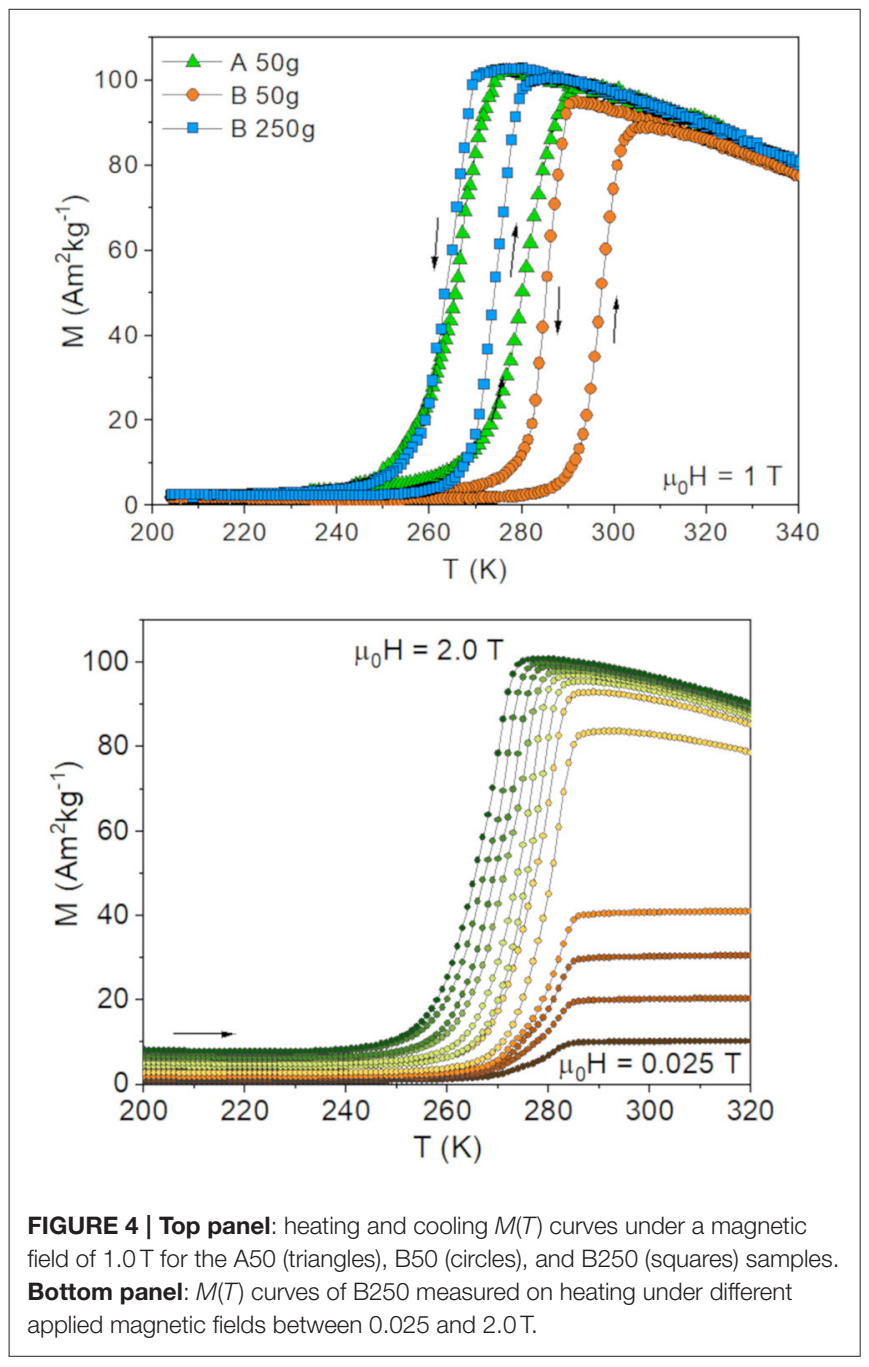

by a large value of magnetization. The magnetization jump at the transition $(\Delta M)$ varies due to the change of transition temperature, and it is between $87 \mathrm{Am}^{2} \mathrm{~kg}^{-1}$ (sample B50, transition on heating) and $100 \mathrm{Am}^{2} \mathrm{~kg}^{-1}$ (sample B250, transition on cooling). The first-order transition temperature is comparable with the results of Gottschall et al. (2015), and it is lower than the data of Liu et al. (2012) due to the lower Co content. In addition, the transition temperature slightly varies among the three samples due to the small changes in the obtained composition. The shift of the critical temperatures is affected by the changes of all the chemical species, and it is consistent with

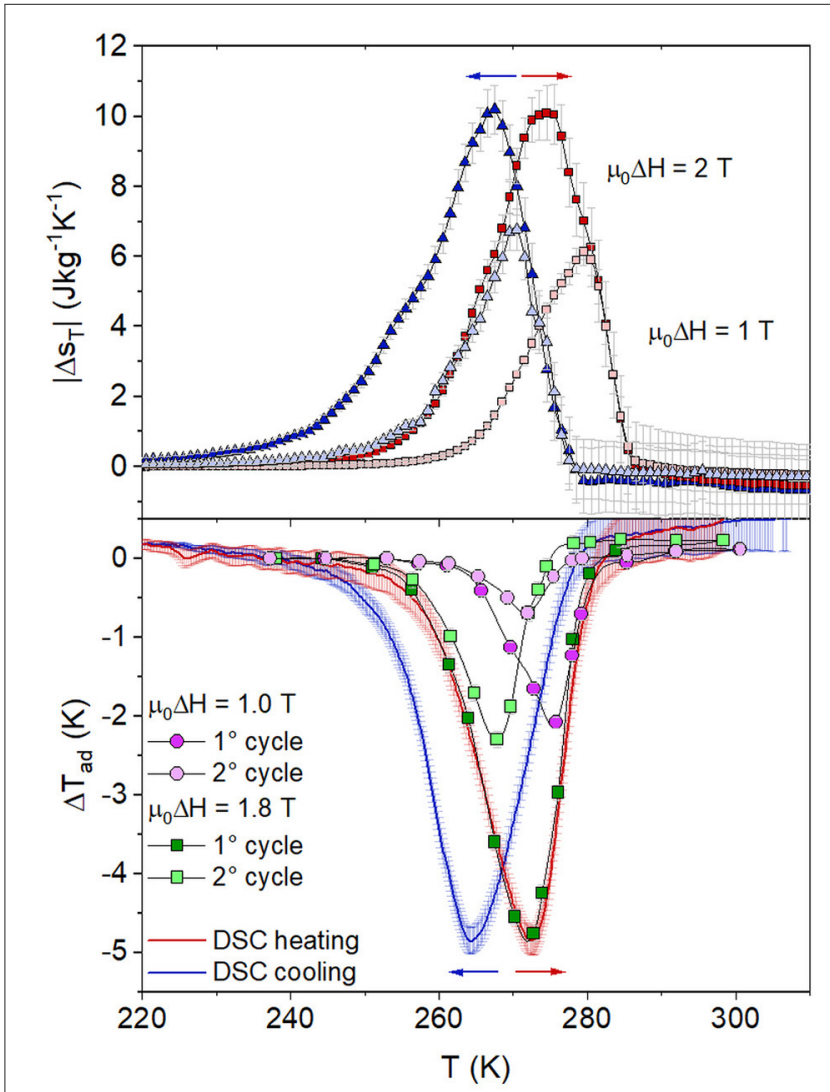

FIGURE 5 | Top panel: $\Delta s_{T}(T)$ of the B250 sample for a magnetic field change of 2.0T (filled symbols) and 1.0T (empty symbols) on heating (red squares) and on cooling (blue triangles). Bottom panel: $\Delta T_{\text {ad }}(T)$ of the B250 induced by applying in the first and second cycles a magnetic field of $1.0 \mathrm{~T}$ (circles) and 1.8T (squares) and derived from in-field differential scanning calorimeter (DSC) measurements (red and blue lines) for a $\mu_{0} \Delta H$ of 1.8T.

the findings already reported in the literature for series of samples with compositions close to the ones studied here (Gottschall et al., 2016). By changing the synthesis protocol, the thermal hysteresis of the martensitic transition is reduced from $14 \pm 1 \mathrm{~K}$ of sample A to $11 \pm 1 \mathrm{~K}$ of $\mathrm{B} 50$ and $10 \pm 1 \mathrm{~K}$ of B250, a feature that promotes a larger reversible MCE in group B samples. Sample B250 shows also the smallest width of the transition, which is an important result to reduce the magnetic field required to fully induce the magnetostructural transition (Cugini et al., 2016). This enhancement is probably due to an improvement of the homogeneity and microstructure of the compound (Cugini et al., 2017) as evidenced also from the mechanical characterization. 
The bottom panel of Figure 4 reports the temperature dependence of magnetization of sample B250 measured on heating under different applied magnetic field, from 0.025 to $2 \mathrm{~T}$. The magnetic field shifts the structural transition to lower temperatures, by promoting the high-moment austenitic phase. The field rate of transition temperature shift is $\Delta T / \mu_{0} \Delta H=8 \pm$ $1 \mathrm{~K} / \mathrm{T}$. This value is comparable to the result of Gottschall et al. (2015), and it is larger than data reported for Heusler compounds with a similar composition (Liu et al., 2012; Li et al., 2019).

The magnetocaloric effect, measured both as adiabatic temperature change $\left(\Delta T_{\mathrm{ad}}\right)$ and isothermal entropy change $\left(\Delta s_{T}\right)$, of sample B250 was completely characterized as a function of temperature and field change by direct and indirect methods (Porcari et al., 2012). The $\Delta s_{T}$ was derived by applying the Maxwell relation at $M(T)$ data, which were measured at different applied magnetic fields with temperature sweeps on cooling and on heating across the magnetostructural transformation. To avoid errors due to the thermal hysteresis, the "iso-field" protocol reported in Caron et al. (2017) was adopted. The experimental error propagation due to the numerical elaboration of data was performed by following the method described in Pecharsky and Gschneidner (1999). The measured values are consistent with the best results reported in the literature for this compound. The top panel of Figure 5 reports the absolute value of $\Delta s_{T}$ as a function of temperature for the transition on cooling and on heating for a magnetic field change of 1 and $2 \mathrm{~T}$. The maximum value of $\Delta s_{T}$ for a magnetic field change of $2 \mathrm{~T}$ is $10.1 \pm 0.8 \mathrm{~J}$ $\mathrm{kg}^{-1} \mathrm{~K}^{-1}$. This value is slightly less than the result reported in Gottschall et al. (2016). The superposition of the cooling and heating $\Delta s_{T}(T)$ curves suggests the exploitability of a reversible MCE in a temperature range $\sim 270 \mathrm{~K}$.

The adiabatic temperature change was directly measured by a homemade experimental setup based on a Cernox bare chip temperature sensor [details on the instrument can be found in Porcari et al. (2013)]. Considering the large thermal hysteresis, a "phase-reset" protocol was utilized, similar to the protocol

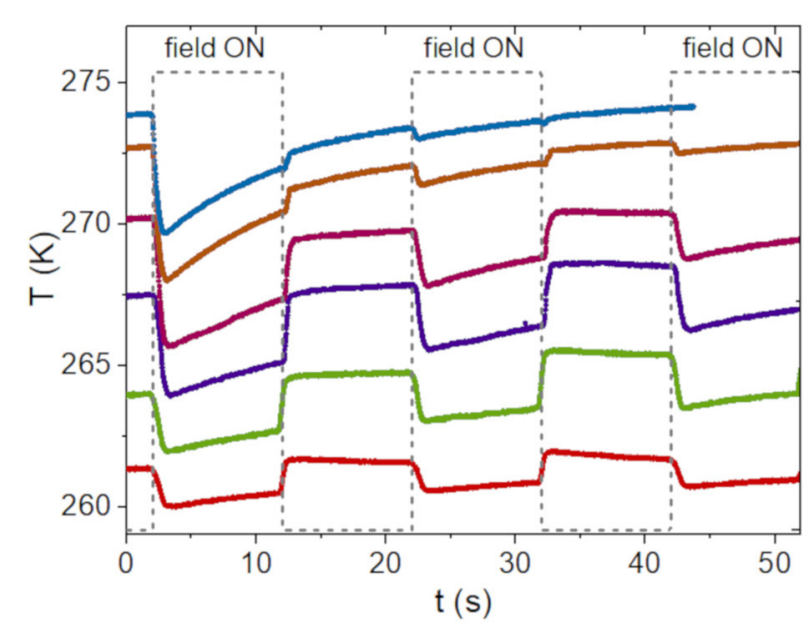

FIGURE 6 | Temperature variation of the B250 sample induced by subsequent application and removal of a 1.8-T magnetic field. suggested to perform $M(H)$ measurements for the calculation of the $\Delta s_{T}$ (Caron et al., 2017), to measure the maximum adiabatic temperature change $\Delta T_{\mathrm{ad}}$. Before each measurement, the sample was cooled down to $200 \mathrm{~K}$ under zero field, to ensure a fully martensitic state. Then, the sample was heated under zero field to the measurement temperature with a sweeping rate of $2 \mathrm{~K} / \mathrm{min}$. As soon as the desired temperature has been reached, a magnetic field of 1 or $1.8 \mathrm{~T}$ was applied to the sample. A total of three cycles of application and removal of the magnetic field were subsequently repeated to measure the first (irreversible) temperature change and the cyclable (reversible) variation. Figure 6 reports some examples of measurements performed at different temperatures with a magnetic field change of $1.8 \mathrm{~T}$. The temperature of the sample is reported as a function of time during three subsequent application and removal of the magnetic field. The measurements performed at lower temperatures show an almost fully reversible MCE: the absolute value of the temperature change is almost the same for all the application (negative $\Delta T_{\mathrm{ad}}$ ) and removal of the magnetic field (positive $\Delta T_{\mathrm{ad}}$ ). By increasing the starting temperature of the measurement, the reversible part of the MCE disappears because of the thermal hysteresis. After the first negative change in temperature, induced by the first application of the magnetic field at $2 \mathrm{~s}$, the following changes of magnetic field do not induce almost any effect on the sample. The maximum reversible effect $(-2.3 \pm 0.1 \mathrm{~K})$ appears at $268 \pm 1 \mathrm{~K}$, in agreement with entropy change results. The reversible effect is about $50 \%$ of the maximum adiabatic temperature change induced with the first field application $(-4.8 \pm 1 \mathrm{~K})$. The bottom panel of Figure 5 summarizes the temperature dependence of the $\Delta T_{\text {ad }}$ obtained by a magnetic field change of 1.0 and $1.8 \mathrm{~T}$ for the first and second variation. The $\Delta T_{\text {ad }}(T)$ values measured directly have been compared also to the ones that can be derived from infield differential scanning calorimeter measurements performed

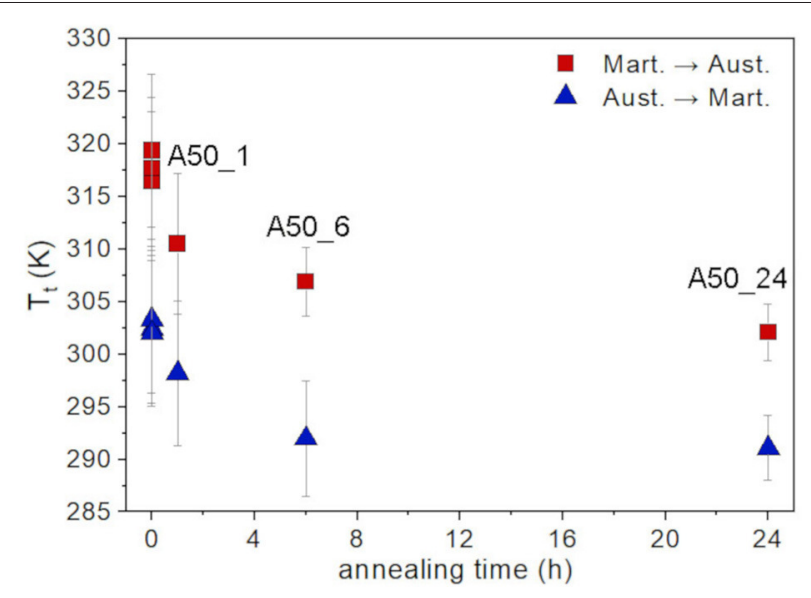

FIGURE 7 | Heating (red squares) and cooling (blue triangles) transition temperatures for fragments of the A50 sample subjected to heat treatments at $523 \mathrm{~K}$, reported as a function of the annealing time $(0,1,6$, and $24 \mathrm{~h})$. The error bar corresponds to the width at half maximum of the Gaussian function used to fit the distribution of the transition with temperature. The data are derived from $M(T)$ measurements performed under an applied magnetic field of $1 \mathrm{~T}$. 


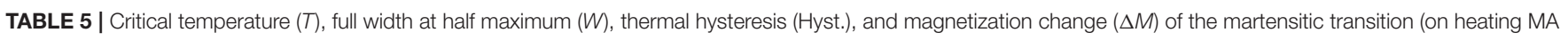
and on cooling AM) of three fragments of the A50 sample before and after an annealing treatment at 523 K for 1 h (A50_1), 6h (A50_6), and 24 h (A50_24), respectively.

\begin{tabular}{|c|c|c|c|c|c|c|c|c|}
\hline & & $\begin{array}{c}\boldsymbol{T}_{\mathrm{MA}} \\
\mathbf{K}\end{array}$ & $\begin{array}{c}T_{\text {AM }} \\
\mathbf{K}\end{array}$ & $\begin{array}{c}W_{\mathrm{MA}} \\
\mathrm{K}\end{array}$ & $\begin{array}{c}W_{\mathrm{AM}} \\
\mathrm{K}\end{array}$ & $\begin{array}{c}\text { Hyst. } \\
\text { K }\end{array}$ & $\begin{array}{c}\Delta M_{\mathrm{MA}} \\
\mathrm{Am}^{2} \mathrm{~kg}^{-1}\end{array}$ & $\begin{array}{c}\Delta M_{\mathrm{AM}} \\
\mathrm{Am}^{2} \mathrm{~kg}^{-1}\end{array}$ \\
\hline \multirow{3}{*}{$\begin{array}{l}\text { Before } \\
\text { Ann. }\end{array}$} & $\mathrm{A} 50^{0}{ }_{-} 1$ & 316.5 & 302.4 & 13.2 & 14.1 & 14.1 & 83 & 87 \\
\hline & A50 $0^{\circ} 6$ & 317.7 & 303.3 & 13.4 & 13.9 & 14.4 & 83 & 87 \\
\hline & $\mathrm{A} 50^{\circ} \_24$ & 319.4 & 302 & 14.5 & 13.8 & 17.4 & 81 & 84 \\
\hline \multirow{3}{*}{$\begin{array}{l}\text { After } \\
\text { Ann. }\end{array}$} & A50_1 & 310.5 & 298.2 & 13.4 & 13.8 & 12.3 & 87 & 90 \\
\hline & A50_6 & 306.9 & 292.0 & 6.5 & 11 & 14.9 & 90 & 93 \\
\hline & A50_24 & 302.1 & 291.1 & 5.4 & 6.2 & 11.0 & 90 & 94 \\
\hline
\end{tabular}

All data are derived from $M(T)$ measurements under an applied magnetic field of $1 \mathrm{~T}$. The untreated samples are marked with a 0 superscript.

on heating and on cooling with 0 and $1.8 \mathrm{~T}$ applied magnetic field. The agreement between the two methods is very high, as expected from previous studies (Porcari et al., 2012). By displaying both the heating and cooling curves, it is possible to appreciate the effect of the transformation hysteresis on the magnetocaloric response of this material. The obtained $\Delta T_{\text {ad }}$ values are slightly lower compared to results reported in the literature (Liu et al., 2012; Gottschall et al., 2015) yet have been obtained on a sample of much greater mass.

\section{Effects of the Low-Temperature Treatments}

The impact of a final low temperature thermal treatment performed on sample A50 are presented in Figure 7 and Table 5. The heating $(\mathrm{M}-\mathrm{A})$ and cooling $(\mathrm{A}-\mathrm{M})$ transition temperatures are reported as a function of the annealing time for three fragments of the sample A50 (A50_1, A50_6, and A50_24), which were treated at $523 \mathrm{~K}$ for 1,6 , and $24 \mathrm{~h}$, respectively, as described in Materials and Methods and in Table 2. It is possible to appreciate the degree of homogeneity of the starting A50 sample by observing the transition temperatures of the different fragments before the annealing (plotted in Figure 7 at annealing time $=0$ ): all the data are consistent considering the experimental error. In addition, the progressive effect of increasing the annealing time is evident: the critical temperatures shift toward slightly lower temperatures, and, most importantly, the hysteresis reduces by $37 \%$, from $17.4 \mathrm{~K}$ of the untreated A50 sample to $11 \mathrm{~K}$ of the sample A50_24 annealed for $24 \mathrm{~h}$. Furthermore, the broadening of the transition is also reduced by increasing the annealing time, anticipating positive effects on the magnetocaloric performances as demonstrated in the literature (Cugini et al., 2016): the possible application of low temperature treatments for the reduction in hysteresis and transition width is a promising route for improving the reversibility of the magnetocaloric effect.

\section{CONCLUSIONS}

A reliable method for synthesizing large quantities of magnetocaloric Heusler compounds has been tested in batches up to $250 \mathrm{~g}$ and provided homogeneous alloys with well-defined composition. The method here employed shows potential for further upscaling. We have demonstrated the possibility to obtain Heusler compounds with remarkable and homogeneous magnetocaloric properties starting from industrial grade reagents, which are consistently cheaper than laboratory ones. Using compacted powders, it is easier to achieve homogeneous melting at lower temperatures and for consistently lower melting times when compared to standard melting of reagents as bulk pieces. Furthermore, mixing by ball milling can be conveniently scaled up to industrially relevant large quantities. A complete magnetocaloric characterization proved that the materials produced show magnetocaloric properties comparable to the best results available in the literature for the same material. A series of thermal treatments proved effective in improving transition sharpness and reversibility of the magnetocaloric effect. The overall results as well as the equipment and reagents employed suggest that this method is promising for further scalability.

\section{DATA AVAILABILITY STATEMENT}

The datasets generated for this study are available on request to the corresponding author.

\section{AUTHOR CONTRIBUTIONS}

FP and VM developed the synthesis protocols, fabricated the samples, and performed mechanical and compositional analysis. FC, NS, and CB performed the magnetic and magnetocaloric measurements and data analysis, as well as on some of the thermal treatments. SF performed the structural characterization, supervised the project in which this research was conducted, and finalized the manuscript. MS and FA supervised all the aspects of the research and the data analysis and interpretation. All authors analyzed and discussed the results and contributed to the writing of this manuscript.

\section{FUNDING}

This work has been supported by the FRIMAG project, funded by Emilia Romagna region within the 2014-20 POR-FESR program (CUP E32F16000190007). 


\section{REFERENCES}

Aksoy, S., Acet, M., Wassermann, E. F., Krenke, T., Moya, X., Mañosa, L., et al. (2009). Structural properties and magnetic interactions in martensitic Ni-Mn-Sb alloys. Philos. Mag. 89, 2093-2109. doi: 10.1080/14786430903082006

Bruno, N. M., Salas, D., Wang, S., Roshchin, I. V., Santamarta, R., Arroyave, R., et al. (2018). On the microstructural origins of martensitic transformation arrest in a NiCoMnIn magnetic shape memory alloy. Acta Mater. 142, 95-106. doi: 10.1016/j.actamat.2017.08.037

Caron, L., Doan, N. B., and Ranno, L. (2017). On entropy change measurements around first order phase transitions in caloric materials. J. Phys. Condens. Matter 29:075401. doi: 10.1088/1361-648X/aa50d1

Cavazzini, G., Cugini, F., Gruner, M. E., Bennati, C., Righi, L., Fabbrici, S., et al. (2019). Tuning the magnetic and magnetocaloric properties of austenitic Ni-Mn-(In,Sn) Heuslers. Scripta Mater. 170, 48-51. doi: 10.1016/j.scriptamat.2019.05.027

Chen, L., Hu, F. X., Wang, J., Shen, J., Zhang, J., Sun, J. R., et al. (2010). Magnetoresistance and magnetocaloric effect in metamagnetic alloys $\mathrm{Ni}_{45} \mathrm{Co}_{5} \mathrm{Mn}_{36.5} \mathrm{In}_{13.5}$. J. Appl. Phys. 107, 09A940-09A943. doi: $10.1063 / 1.3359806$

Chen, L., Hu, F. X., Wang, J., Zhao, J. L., Sun, J. R., Shen, B. G., et al. (2011). Tuning martensitic transformation and magnetoresistance effect by low temperature annealing in $\mathrm{Ni}_{45} \mathrm{Co}_{5} \mathrm{Mn}_{36.6} \mathrm{In}_{13.4}$ alloys. J. Phys. D Appl. Phys. 44:085002. doi: 10.1088/0022-3727/44/8/085002

Cugini, F., Porcari, G., Fabbrici, S., Albertini, F., and Solzi, M. (2016). Influence of the transition width on the magnetocaloric effect across the magnetostructural transition of Heusler alloys. Philos. Trans. A Math. Phys. Eng. Sci. 374:20150306. doi: 10.1098/rsta.2015.0306

Cugini, F., Porcari, G., Rimoldi, T., Orsi, D., Fabbrici, S., Albertini, F., et al. (2017). On the broadening of the martensitic transition in heusler alloys: from microscopic features to magnetocaloric properties. J. Metals 69, 1422-1426. doi: 10.1007/s11837-017-2373-Z

Fabbrici, S., Porcari, G., Cugini, F., Solzi, M., Kamarad, J., Arnold, Z., et al. (2014). Co and In doped Ni-Mn-Ga magnetic shape memory alloys: a thorough structural, magnetic and magnetocaloric study. Entropy 16, 2204-2222. doi: $10.3390 / \mathrm{e} 16042204$

Feng, Y., Chen, H., Fei, X., Bian, X., and Wang, P. (2018). Improvement of mechanical property and large shape recovery of sintered $\mathrm{Ni}_{45} \mathrm{Mn}_{36.6} \mathrm{In}_{13.4} \mathrm{Co}_{5}$ alloy. J. Alloys Compd. 765, 264-270. doi: 10.1016/j.jallcom.2018.06.244

Feng, Y., Sui, J. H., Gao, Z. Y., Dong, G. F., and Cai, W. (2009). Microstructure, phase transitions and mechanical properties of $\mathrm{Ni}_{50} \mathrm{Mn}_{34} \mathrm{In}_{16-\mathrm{y}} \mathrm{Co}_{\mathrm{y}}$ alloys. J. Alloys Compd. 476, 935-939. doi: 10.1016/j.jallcom.2008.09.149

Franco, V., Blázquez, J. S., Ipus, J. J., Law, J. Y., Moreno-Ramírez, L. M., and Conde, A. (2018). Magnetocaloric effect: from materials research to refrigeration devices. Prog. Mater. Sci. 93, 112-232. doi: 10.1016/j.pmatsci.2017.10.005

Gauss, R., Homm, G., and Gutfleisch, O. (2017). The resource basis of magnetic refrigeration. J. Ind. Ecol. 21, 1291-1300. doi: 10.1111/jiec.12488

Gottschall, T., Skokov, K. P., Benke, D., Gruner, M. E., and Gutfleisch, O. (2016) Contradictory role of the magnetic contribution in inverse magnetocaloric Heusler materials. Phys. Rev. B 93:184431. doi: 10.1103/PhysRevB.93.184431

Gottschall, T., Skokov, K. P., Frincu, B., and Gutfleisch, O. (2015). Large reversible magnetocaloric effect in Ni-Mn-In-Co. Appl. Phys. Lett. 106:021901. doi: $10.1063 / 1.4905371$

International Institute on Refrigeration IIR_IIF (2019). 38th Informatory Note on Refrigeration Technology. Publications Informatory Notes. Available online at: http://www.iifiir.org/ (accessed October 31, 2019).

Ito, K., Ito, W., Umetsu, R. Y., Tajima, S., Kawaura, H., Kainuma, R., et al. (2009). Metamagnetic shape memory effect in polycrystalline NiCoMnSn alloy fabricated by spark plasma sintering. Scr. Mater. 61, 504-507. doi: 10.1016/j.scriptamat.2009.05.008
Ito, W., Nagasako, M., Umetsu, R. Y., Kainuma, R., Kanomata, T., and Ishida, K. (2008). Atomic ordering and magnetic properties in the $\mathrm{Ni}_{45} \mathrm{Co}_{5} \mathrm{Mn}_{36.7} \mathrm{In}_{13.3}$ metamagnetic shape memory alloy. Appl. Phys. Lett. 93:232503. doi: 10.1063/1.3043456

Kainuma, R., Imano, Y., Ito, W., Sutou, Y., Morito, H., Okamoto, S., et al. (2006). Magnetic-field-induced shape recovery by reverse phase transformation. Nature 439, 957-960. doi: 10.1038/nature04493

Kitanovski, A., Tušek, J., Tomc, U., Plaznik, U., OŽbolt, M., and Poredo,š M. (2015). Magnetocaloric Energy Conversion. Springer International Publishing.

Li, Z., Yang, J., Li, D., Li, Z., Yang, B., Yan, H., et al. (2019). Tuning the reversible magnetocaloric effect in Ni-Mn-In-based alloys through Co and Cu Co-doping. Adv. Electron. Mater. 5:1800845. doi: 10.1002/aelm.201 800845

Liu, J., Gottschall, T., Skokov, K. P., Moore, J. D., and Gutfleisch, O. (2012). Giant magnetocaloric effect driven by structural transitions. Nat. Mater. 11, 620-626. doi: $10.1038 /$ nmat3334

Pareti, L., Solzi, M., Albertini, F., and Paoluzi, A. (2003). Giant entropy change at the co-occurrence of structural and magnetic transitions in the $\mathrm{Ni}_{2.19} \mathrm{Mn}_{0.81} \mathrm{Ga}$ Heusler alloy. Eur. Phys. J. B 32, 303-307. doi: 10.1140/epjb/e2003-00102-y

Pecharsky, V. K., and Gschneidner, K. A. (1997). Giant magnetocaloric effect in $\mathrm{Gd}_{5}\left(\mathrm{Si}_{2} \mathrm{Ge}_{2}\right)$. Phys. Rev. Lett. 78, 4494-4497. doi: 10.1103/PhysRevLett.78.4494

Pecharsky, V. K., and Gschneidner, K. A. Jr. (1999). Magnetocaloric effect from indirect measurements: magnetization and heat capacity. J. Appl. Phys. 86, 565-575. doi: 10.1063/1.370767

Porcari, G., Buzzi, M., Cugini, F., Pellicelli, R., Pernechele, C., Caron, L., et al. (2013). Direct magnetocaloric characterization and simulation of thermomagnetic cycles. Rev. Sci. Instrum. 84:073907. doi: 10.1063/1.4815825

Porcari, G., Cugini, F., Fabbrici, S., Pernechele, C., Albertini, F., Buzzi, M., et al. (2012). Convergence of direct and indirect methods in the magnetocaloric study of first order transformations: the case of Ni-Co-Mn-Ga Heusler alloys. Phys. Rev. B 86:104432. doi: 10.1103/PhysRevB.86.104432

Righi, L., Albertini, F., Fabbrici, S., and Paoluzi, A. (2011). Crystal structures of modulated martensitic phases of FSM heusler alloys. Mater. Sci. Forum 684, 105-116. doi: 10.4028/www.scientific.net/MSF.684.105

Sánchez-Alarcos, V., Pérez-Landazábal, J. I., Recarte, V., Gómez-Polo, C., and Rodríguez-Velamazán, J. A. (2008). Correlation between composition and phase transformation temperatures in Ni-Mn-Ga-Co ferromagnetic shape memory alloys. Acta Mater. 56, 5370-5376. doi: 10.1016/j.actamat.2008.07.017

Trombi, L., Cugini, F., Rosa, R., Sarzi Amadè, N., Chicco, S., Solzi, M., et al., (2020). Rapid microwave synthesis of magnetocaloric Ni-Mn-Sn Heusler compounds. Scripta Mater. 176, 63-66. doi: 10.1016/j.scriptamat.2019.09.039

Waske, A., Gruner, M. E., Gottschall, T., and Gutfleisch, O. (2018). Magnetocaloric materials for refrigeration near room temperature. MRS Bull. 43, 269-273. doi: $10.1557 / \mathrm{mrs} .2018 .69$

Zimm, C., Jastrab, A., Sternberg, A., Pecharsky, V., Gschneidner, K. Jr., et al. (1998). Description and performance of a near room temperature magnetic refrigerator. Adv. Cryog. Eng. 43, 1759-1766. doi: 10.1007/978-1-4757-9047-4_222

Conflict of Interest: The authors declare that the research was conducted in the absence of any commercial or financial relationships that could be construed as a potential conflict of interest.

Copyright (C) 2020 Puglielli, Mussi, Cugini, Sarzi Amadè, Solzi, Bennati, Fabbrici and Albertini. This is an open-access article distributed under the terms of the Creative Commons Attribution License (CC BY). The use, distribution or reproduction in other forums is permitted, provided the original author(s) and the copyright owner(s) are credited and that the original publication in this journal is cited, in accordance with accepted academic practice. No use, distribution or reproduction is permitted which does not comply with these terms. 